\title{
July 2018 Arizona Thoracic Society Notes
}

The July 2018 Arizona Thoracic Society meeting was held on Wednesday, July 25, 2018 at the HonorHealth Rehabilitation Hospital beginning at 6:30 PM. This was a dinner meeting with case presentations. There were 8 in attendance representing the pulmonary, critical care, sleep, and radiology communities.

At the beginning of the meeting several issues were discussed:

- The dwindling attendance at these meetings. It was decided to again reach out to the pulmonary fellowship programs at Mayo, University of Arizona Phoenix and University of Arizona Tucson.

- The two recent cases of pharmacists refusing to dispense medications was discussed without coming to a consensus. One physician said he would refuse to submit prescriptions to the Peoria Walgreens.

- Discussion regarding the Tobacco 21 which had been killed in committee by Rep. Jeff Weninger was tabled until the next meeting.

There were 2 case presentations:

1. Dr. Lewis Wesselius presented a 67-year-old woman who had increasing shortness of breath. She was started on prednisone by another physician and her dyspnea improved although it recurred when her prednisone was tapered. Unfortunately, she gained $30 \mathrm{lbs}$. while on the prednisone. The patient did not smoke. She had a few crackles at her right base but otherwise the physical examination was normal. She desaturated with exercise. Her CT scan showed ground glass opacities with multiple small cysts. PFTs were restrictive with a DLCO of $55 \%$ of predicted. She was further questioned about additional symptoms and stated she had dry mouth and eyes for years. Rheumatology consultation was ordered, her SSP and SSA were both positive, and she had a diagnosis of Sjogren's syndrome made. Because she was symptomatic it was thought she needed to treatment but she did not want to take prednisone again. For this reason, she was begun on mycophenolate.

2. Dr. Gerald Schwartzberg presented a 62-year-old man who was seen in the office with shortness of breath. Physical examination was normal and he had normal PFTs. He was admitted to the hospital a couple of weeks later after a trip with a pulmonary embolus. Discussion centered on when a CT angiogram should be ordered. There was a consensus that a CTangiogram did not need to be done in all patients complaining of dyspnea but no consensus on criteria for whom it should be ordered.

The meeting was adjourned about 8 PM. The next meeting will be on September 26 at 6:30 PM at HonorHealth Rehabilitation Hospital.

Richard A. Robbins MD

Editor, SWJPCC 